\title{
CINÉTICA DE SECAGEM DO MESOCARPO DE BACURI
}

\author{
L.J.S. SILVA ${ }^{1}$, D.C. SILVA ${ }^{1}$, I.A. LOPES ${ }^{1}$, H.A. VILLA-VÉLEZ ${ }^{1}$ e A.A. SANTANA ${ }^{1}$ \\ ${ }^{1}$ UFMA - Universidade Federal do Maranhão, Centro de Ciências Exatas e Tecnologia, Faculdade \\ de Engenharia Química \\ E-mail para contato: layrtonself@gmail.com
}

\begin{abstract}
RESUMO - O bacuri (Platonia insignis) é um fruto tradicional das regiões Norte e Nordeste do Brasil, conhecido por suas propriedades nutricionais, sabor e odor característicos, além das propriedades medicinais de seus constituintes que são alvos de pesquisa científica. Entre os processos para sua industrialização, a secagem é uma das operações unitárias que mais precisam de pesquisa, sendo está operação que proporciona informações sobre as melhores condições de armazenamento e as características físico-químicas finais do produto seco. Assim, o objetivo do trabalho é modelar as curvas de secagem de polpa de Bacuri obtidas em uma estufa, nas temperaturas de 50 e $60^{\circ} \mathrm{C}$. Para isto, foram empregados os modelos empirícos de Weibull, Peleg, Henderson-Pabis, Lewis e Page, através de uma regressão não linear. Todos os ajustes apresentaram coeficiente de determinação $\left(R^{2}\right)$ superior à 0,910 , com o modelo de Page apresentando o melhor ajuste. Além disso, foi observado o efeito da elevação da temperatura no aumento da velocidade de secagem para as curvas de 50 e $60{ }^{\circ} \mathrm{C}$.
\end{abstract}

\section{INTRODUÇÃO}

O bacurizeiro pertence à família Clusiaceae, subfamília Clusioideae e ao gênero Platonia, que é monotipo. A família botânica Clusiaceae engloba aproximadamente 1000 espécies subordinadas a 47 gêneros, dispersos em regiões tropicais e subtropicais do mundo (Barroso et al., 2002; Barbosa et al., 1978; Brummit, 1992; Cronquist, 1981).

O seu fruto, é uma baga grande de forma ovóide ou quase circular, com diâmetro médio de $8 \mathrm{~cm}$ e comprimento de $7 \mathrm{~cm}$. O peso varia de 100 a $500 \mathrm{~g}$, sendo $70 \%$ de casca, $18 \%$ de semente e apenas $18 \%$ de polpa. O mesocarpo (ou casca), que constitui a maior porção do fruto, apresenta sabor e odor semelhantes ao da polpa e, portanto, com excelentes qualidades para aproveitamento na fabricação de doces e refrescos (Barbosa et al., 1978). Entretanto, seu aproveitamento não tem ocorrido devido à forte presença da resina (Mourão e Beltrati, 1995).

Devido ao grande potencial deste fruto é importante preservar os diferentes componentes presentes que se encontram-se misturados com a água interna no material. A aplicação de métodos de conservação pode oferecer vantagens importantes na reutilização e no tratamento destes produtos. Atualmente, com o avanço da tecnologia, estes procedimentos têm sido na sua maioria trocados por processos mais eficientes e controlados, tais como secadores de convecção de ar quente. A secagem é um processo complexo onde os fenômenos simultâneos de transferência de calor e massa contribuem para a remoção de umidade, levando a reduções substanciais na massa do produto e do volume, assim, como minimizar os custos de embalagem, armazenamento e transporte (Vega-Gálvez et al., 2010). 
Para o processo de secagem, a relação que existe entre o conteúdo de umidade de equilíbrio e a atividade de água dos alimentos é amplamente pesquisada (Mulet et al., 2002). A modelagem do processo de secagem traz conhecimentos matemáticos e físicos no processo, e muitos estudos têm-se dedicado a analisar os diferentes aspectos deste fenómeno. Alguns estudos publicados na literatura incluem um estudo da distribuição do perfil de difusividade e o encolhimento sob condições de ar forçado (Vega-Gálvez et al., 2010; Clemente et al., 2011), o efeito das resistências internas e externas na transferência de massa e calor com alto teor de umidade (Bartzanas et al., 2010; Smolka et al., 2010), e a dependência das propriedades físicas e de transporte sobre a temperatura dos alimentos e o teor de umidade (Askari et al., 2009; Bon et al., 2010).

Assim, o seguinte trabalho tem como objetivo estudar as cinéticas de secagem da polpa de Bacuri em função da temperatura.

\section{MATERIAIS E METODOS}

\subsection{Matéria-prima}

Neste trabalho foram utilizados bacuris da espécie Platonia insignis Mart., obtidos no mercado local da cidade de Imperatriz - MA. Todos os frutos foram recolhidos após terem chegado à maturação. Foram selecionados os frutos de aparência mais uniforme para a padronização das amostras sendo que frutos com muitas deformações e manchas foram descartados.

\subsection{Cinética de Secagem}

O primeiro passo para a realização da cinética de secagem foi a definição da geometria que seria utilizada para se realizar o experimento. A determinação do formato de um paralelepípedo com dimensões de $1 \mathrm{~cm}$ de largura, $1 \mathrm{~cm}$ de comprimento e $0,5 \mathrm{~cm}$ de espessura. Esta geometria foi escolhida com o objetivo de verificar a secagem em um pequeno elemento do mesocarpo em um menor tempo.

A secagem do mesocarpo foi realizada em amostras de aproximadamente $4 \mathrm{~g}$ por meio de uma estufa convectiva nas temperaturas de 50 e $60{ }^{\circ} \mathrm{C}$. Foram previamente escolhidos intervalos apropriados para a realização das pesagens do material, sendo que as pesagens foram executadas rapidamente para evitar variações bruscas na temperatura da estufa e aquisição de umidade pelo mesocarpo durante a secagem, evitando assim erros durante a análise dos resultados e estudo da cinética de secagem. Após cada experimentação, as curvas de secagem de Bacuri foram expressas em conteúdo de umidade em base seca (b.s.), através da relação com o conteúdo de umidade inicial da amostra.

\subsection{Modelagem matemática}

Para os ajustes dos resultados utilizou-se cinco modelos semi-empíricos disponíveis na literatura para secagem de camada delgada, o modelo de Weibull (Equação 1), modelo de Peleg (Equação 2), modelo de Henderson-Pabis (Equação 3), modelo de Lewis (Equação 4) e o modelo de Page (Equação 5). Com o objetivo de determinar qual ofereceria uma descrição mais adequada do fenômeno analisado. A qualidade do ajuste seria avaliada através do 
coeficiente de determinação $R^{2}$ e do valor do erro médio relativo (MRE) que está descrito na Equação 6.

$$
\begin{aligned}
& X=X_{e}+\left(X_{o}-X_{e}\right) \exp \left(\frac{-t}{\beta}\right) \\
& X=X_{o}-\frac{t}{k_{1}+k_{2} t} \\
& X=X_{e}+\left(X_{o}-X_{e}\right) a \exp (-k t) \\
& X=X_{e}+\left(X_{o}-X_{e}\right) \exp (-k t) \\
& X=X_{e}+\left(X_{o}-X_{e}\right) a \exp \left(-k t^{N}\right)
\end{aligned}
$$

onde, $X_{e}$ e $X_{\mathrm{o}}$ são as umidades de equilíbrio e a umidade inicial, respectivamente. $k_{1}, k_{2}, a, \beta$ e $N$ são os parâmetros de ajuste dos modelos matemáticos. $\mathrm{O}$ coeficiente de determinação $\mathrm{R}^{2}$ entre as respostas e os valores preditos e o valor do erro relativo médio $M R E$ descrito pela Equação 6.

$$
\operatorname{MRE}(\%)=\frac{100}{n} \sum_{i=1}^{n} \frac{|(M i-M p i)|}{M i}
$$

em que $M_{i}$ é o valor experimental, $M p i$ é o valor predito e, $n$ é o número de dados experimentais.

\section{RESULTADOS E DISCUSSÃO}

\subsection{Cinética de Secagem}

Na Tabela 1 estão representados os valores obtidos para os parâmetros e o valor do erro médio relativo e coeficiente de correlação. Ao analisar o conjunto de dados obtido nota-se que todos os modelos semi-empíricos, ajustados por regressão não-linear, descrevem com grande precisão a cinética da secagem. Sendo apresentados erros mínimo e excelentes coeficientes de correlação, todos estão muito próximos do valor 1, o valor máximo deste parâmetro estatístico que representa uma correlação ótima entre o conjunto de dados experimentais e o ajuste executado no modelo. 
Tabela 1 - Resultados estatísticos de diferentes modelos de camada delgada.

\begin{tabular}{|c|c|c|c|}
\hline Modelos & Parâmetros & $\mathbf{5 0}^{\mathbf{0}} \mathbf{C}$ & $\mathbf{6 0}^{\mathbf{}} \mathbf{C}$ \\
\hline \multirow{4}{*}{ Weibull } & $\beta$ & 3724,964 & 2875,236 \\
\cline { 2 - 4 } & $R^{2}$ & 0,994 & 0,995 \\
\cline { 2 - 4 } & $M R E(\%)$ & 19,397 & 8,249 \\
\hline \multirow{4}{*}{ Peleg } & $k_{1}$ & 509,999 & 207,675 \\
\cline { 2 - 4 } & $k_{2}$ & 0,297 & 0,312 \\
\cline { 2 - 4 } & $R^{2}$ & 0,945 & 0,910 \\
\cline { 2 - 4 } & $M R E(\%)$ & 48,336 & 30,353 \\
\hline \multirow{4}{*}{ Henderson-Pabis } & $a$ & 1,000 & 0,885 \\
\cline { 2 - 4 } & $k$ & $2,69 \times 10^{-4}$ & $3,33 \times 10^{-4}$ \\
\cline { 2 - 4 } & $R^{2}$ & 0,994 & 0,994 \\
\cline { 2 - 4 } & $M R E(\%)$ & 19,397 & 6,300 \\
\hline \multirow{4}{*}{ Lewis } & $k$ & $2,690 \times 10^{-4}$ & $3,480 \times 10^{-4}$ \\
\cline { 2 - 4 } & $R^{2}$ & 0,994 & 0,996 \\
\cline { 2 - 4 } & $M R E(\%)$ & 19,401 & 8,252 \\
\hline \multirow{5}{*}{ Page } & $k$ & $7,378 \times 10^{-4}$ & $8,403 \times 10^{-4}$ \\
\cline { 2 - 4 } & $N$ & 1,13 & 0,90 \\
\cline { 2 - 4 } & $R^{2}$ & 0,999 & 0,999 \\
\cline { 2 - 4 } & $M R E(\%)$ & 10,569 & 5,084 \\
\hline
\end{tabular}

Na Figura 1 verifica-se o modelo de Page foi o que apresentou melhor ajuste, com os maiores valores do coeficiente de correlação, beirando a correlação ótima para ambas as temperaturas, e entre os menores erros relativos. Além disso, observou-se através da análise do ajuste de Page que o parâmetro $k$ que indica a constante da taxa de secagem aumentou com o aumento da temperatura, o qual foi constatado por trabalhos feitos de Alexandre et al. (2013). Também se nota pelos pontos experimentais que o aumento da temperatura causou um aumento na taxa de secagem, ou seja, o mesocarpo de bacuri secou mais rapidamente com o aumento da temperatura, fato que apresenta concordância com outros trabalhos disponíveis na literatura, como Morais et al. (2013), Alexandre et al. (2013) e Akpinar et al. (2003). 
Figura 1 - Ajuste experimental da cinética Modelo de Page.

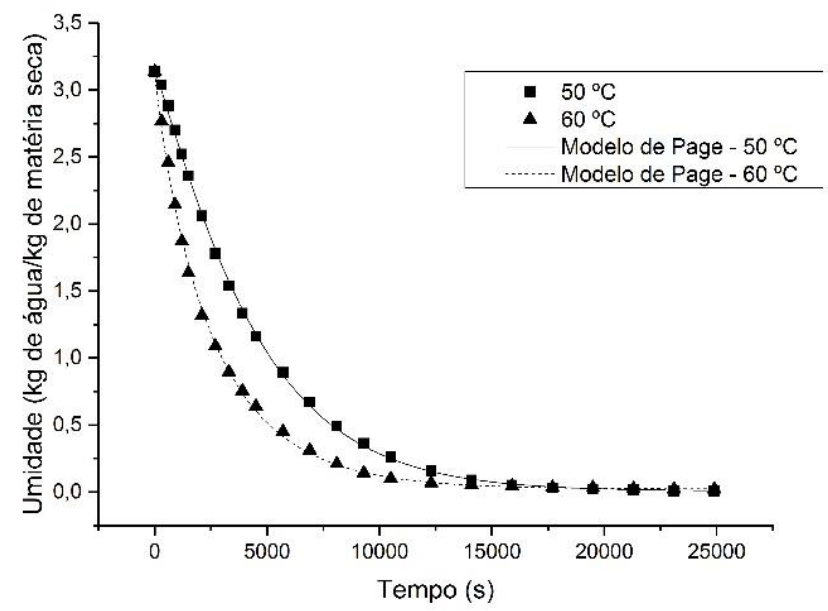

\section{CONCLUSÃO}

Foram obtidas as cinéticas de secagem do bacuri em função das temperaturas selecionadas. A análise da secagem de camada delgada, processo que possui ambas transferências de calor e de massa, demonstrou que ambas as temperaturas são suficientes para realizar uma boa secagem do mesocarpo de bacuri, e que os modelos empíricos disponíveis na literatura descrevem com excelente qualidade os resultados experimentais, principalmente o modelo de Page com ótimo valores de $R^{2}$.

\section{REFERÊNCIAS BIBLIOGRÁFICAS}

AKPINAR, E. K., BICER Y., YILDZ C., Thin layer drying of red pepper, J. of Food Eng., v. 59, p. 99-104, 2003.

ALEXANDRE, Hofsky V. et al. Cinética de secagem do resíduo de abacaxi enriquecido. Rev. Bras. Eng. Agríc. Ambient., Campina Grande , v. 17, p. 640-646, 2013.

ASKARI, G. R.; EMAM-DJOMEH, Z.; MOUSAVI, S. M. An investigation of the effects of drying methods and conditions on drying characteristics and quality attributes of agricultural products during hot air and hot air/microwave-assisted dehydration. Dry. Technol., v. 27, p. 831-841, 2009.

BARBOSA, W.C.; NAZARÉ, R.F.R. de; NAGATA, I. Estudo tecnológico de frutas da Amazônia. Belém: Embrapa-CPATU, 1978.

BARROSO, G. M.; PEIXOTO, A. L.; ICHASO, C. L. F.; GUIMARÃES, E. F.; COSTA, C. G. Sistemática de angiospermas no Brasil. Viçosa, MG: UFV, 2002.

BARTZANAS, T. et al. A numerical modelling approach for biomass field drying. Biosyst. Eng., v. 106, p. 458-469, 2010. 
BON, J. et al. Thermophysical properties of mango pulp (Mangifera indica L. cv. Tommy Atkins). J. Food Eng., v. 97, p. 563-568, 2010.

BRUMMIT, R. K. Vascular plant families and genera. Londres: Royal Botanic Gardens, Kew, 1992.

CLEMENTE, G. et al. Drying modelling of defrosted pork meat under forced convection conditions. Meat Sci., v. 88, p. 374-378, 2011.

CRONQUIST, A. A integrated system of classification of flowering plants. New York: Columbia Un. Press, 1981.

MORAIS, S. J. da S. et al . Modelagem matemática das curvas de secagem e coeficiente de difusão de grãos de feijão-caupi (Vigna unguiculata (L.) Walp.). Rev. Ciênc. Agron., Fortaleza , v. 44, p. 455-463, 2013.

MOURÃO, K S. M. M.; BELTRATI, C. M. Morfologia dos frutos, sementes e plântulas de Platonia insignis Mart. (Clusiaceae). II. Morfo-anatomia dos frutos e sementes maduros. Acta Amazônica, Manaus, v. 25, p. 33-45, 1995.

MULET, A. et al. Equilibrium isotherms and isoteric heats of morel (Morchela esculenta). J. Food Eng., v. 53, p. 75-81, 2002.

SMOLKA, J.; NOVAK, A. J.; RYBARZ, D. Improved 3-D temperature uniformity in a laboratory drying oven based on experimentally validated CFD computations. J. Food Eng., v. 97, p. 373-383, 2010.

VEGA-GÁLVEZ, A. et al. Effective moisture diffusivity determination and mathematical modelling of the drying curves of the olive-waste cake. Bioresource Technol., v. 101, p. 7265-7270, 2010. 